\title{
IMPLEMENTAÇÃO DE UM SISTEMA PARA O ESCALONAMENTO DE ESPESSADORES DE POLPA
}

\author{
Claudio Schneider ${ }^{\prime}$ \\ Vladmir Alves ${ }^{2}$ \\ Oliver Gobbo ${ }^{2}$ \\ Keila Gonçalves ${ }^{2}$
}

\section{Resumo}

O objetivo deste trabalho é desenvolver uma metodologia acessível para simulação e escalonamento de espessadores de lama. Essa metodologia acessível requer, primeiramente, o desenvolvimento de um aplicativo para o modelamento dos ensaios de sedimentação. O modelo de Wilhelm-Naide para sedimentação foi implementado em um aplicativo prático, com interface gráfica apropriada. Com isso os resultados dos testes de sedimentação podem ser traduzidos em termos de alguns parâmetros. Com esses parâmetros em mãos, o método de Kynch para polpas incompressíveis pode ser aplicado automaticamente. O modelo de Kynch foi implementado no simulador de plantas Modsim ${ }^{\mathrm{TM}}$, e a curva de fluxo é calculada com base nos parâmetros de Wilhelm-Naide obtidos a partir dos testes de sedimentação. A metodologia foi testada com dados reais, obtidos em uma campanha de amostragem realizada em um espessador industrial.

Palavras-chave: Espessadores; Sedimentação; Escalonamento; Simulação.

\section{IMPLEMENTATION OF A SYSTEM FOR SCALING UP THICKENERS}

\begin{abstract}
The objective of this work is to develop a methodology that is accessible and suitable for the simulation and the scale up of thickeners. The methodology requires the development of a graphical user interface for the modeling of sedimentation tests. The Wilhelm-Naide sedimentation model was implemented in a computer program, with an appropriate graphical interface and optimization routine. This allows for the calculation of a few parameters that describe just about any sedimentation test in analytical form. With these parameters on hand, the Kynch method for incompressible slurries can be applied in an automated form. Kynch's model has been implemented in Modsim ${ }^{T M}$ plant-wide simulator, and the flux curve is calculated based on the Wilhelm-Naide parameters obtained from specific sedimentation tests. The methodology was tested with real data obtained in a sampling campaign carried out in an industrial thickener.
\end{abstract}

Key words: Thickener; Sedimentation; Scale up; Simulation.

\section{INTRODUÇÃO}

Vários modelos foram propostos para relacionar a velocidade de sedimentação à concentração de sólidos e estes têm sido utilizados com mais ou menos sucesso para descrever a sedimentação de uma variedade de polpas industriais. É praxe expressar estas equações de modelo em termos da concentração volumétrica de sólidos $\varphi$, expressa em $\mathrm{m}^{3}$ de sólidos $/ \mathrm{m}^{3}$ de polpa. As duas representações de concentração de sólidos são relacionadas por

$$
\mathrm{C}=\rho_{\mathrm{s}} \varphi
$$

onde $\rho_{s}$ é a densidade do sólido.

Uma quantidade grande de experimentos realizados cuidadosamente por Wilhelm e Naide, ${ }^{(1)}$ em uma faixa grande de concentrações mostrou que a velocidade $V$ de sedimentação de polpas floculadas em faixas restritas de concentração segue uma função de potência:

$$
\frac{1}{\mathrm{~V}}=\mathrm{aC}^{\mathrm{b}}
$$

Portanto, uma relação entre a velocidade de sedimentação e concentração para qualquer faixa de concentração segue uma equação relativamente simples:

$$
\frac{\mathrm{v}_{\mathrm{TF}}}{\mathrm{V}}=1+\alpha_{1} \mathrm{C}^{\beta_{1}}+\alpha_{2} \mathrm{C}^{\beta_{2}}+\ldots
$$

com $0<\beta_{1}<\beta_{2}<\ldots$ cada termo domina a expressão em uma faixa limitada de concentrações. Esta equação descreve o comportamento de sedimentação de uma variedade de polpas em faixas largas de concentração $C$. Os valores de $\beta_{i}$ variam de 1,0 até 20,0 ou mais. Normalmente não mais do que dois termos da função potência

\footnotetext{
' Tecnologista Sênior, Centro de Tecnologia Mineral, Ministério da Ciência e da Tecnologia. Av. Pedro Calmon, 900 - Cidade Universitária. 2 I94I908 - Rio de Janeiro, RJ. (claudio@mineraltech.com)

${ }^{2}$ Engenheiro Gerência de Tecnologia, Centro de Desenvolvimento Mineral, Companhia Vale do Rio Doce
} 
são requeridos para descrever a velocidade de sedimentação sobre uma ou duas ordens de magnitude de variação de concentração. $\mathrm{Na}$ Equação $3 v_{\text {TF }}$ representa a velocidade terminal de flocos individuais, quando estes estão separados um do outro e se comportam de forma isolada. $v_{T F}$ é muito difícil de ser medida, e seu valor pode ser estimado juntamente com os valores de $\alpha_{\mathrm{i}}$ e $\beta_{\mathrm{i}}$ a partir de dados de sedimentação cuidadosamente levantados. Este modelo também é útil para polpas não floculadas.

A despeito de sua natureza completamente empírica, a equação estendida de Wilhelm-Naide provê uma descrição versátil e flexível da velocidade de sedimentação. Ela é utilizada como base para o desenvolvimento do sistema para escalonamento de espessadores aqui apresentado, baseado no método de $\mathrm{Kynch}^{(2)}$ para polpas incompressíveis.

Ensaios de sedimentação produzem dados que podem ser modelados com a equação estendida de Wilhelm-Naide. Os ensaios produzem dados da altura da interface de sedimentação versus o tempo de sedimentação. A altura da interface diminui monotonicamente com o tempo de sedimentação. A concentração da polpa para um tempo de sedimentação $t$ pode ser calculada diretamente por:

$$
\mathrm{C}(\mathrm{t})=\frac{\mathrm{C}_{0} \mathrm{H}_{0}}{\mathrm{~h}(\mathrm{t})}
$$

onde $\mathrm{C}_{0}$ é a concentração inicial de polpa $\mathrm{C}(0)$ e $\mathrm{H}_{0}$ é a altura inicial da linha de sedimentação, ou altura inicial da polpa na proveta de teste $\mathrm{H}(0)$. Se houverem dados disponíveis, uma relação completa entre tempo de sedimentação e concentração de polpa pode ser derivada facilmente do teste de sedimentação. A velocidade de sedimentação é dada pela inclinação da curva de sedimentação $h(t)$ em qualquer tempo $t$ correspondente à concentração $c(t)$ calculada pela equação 4 .

O modelo de Wilhelm-Naide é dado em termos de concentração versus velocidade, e este dado não está prontamente disponível a partir do ensaio de sedimentação. $O$ método gráfico correspondente requer a estimativa da inclinação da curva de sedimentação nos pontos medidos. Isto praticamente inviabiliza o método porque estimativas deste tipo são facilmente errôneas, além de trabalhosas. A alternativa aqui proposta consiste em integrar o modelo de Wilhelm-Naide para obter-se uma velocidade média de sedimentação entre a concentração inicial e a concentração desejada:

$$
\bar{V}_{t}=\frac{\int_{C(0)}^{C(t)} V(C(t)) d C}{C(t)-C(0)}
$$

onde $\bar{V}_{t}$ é a velocidade média de sedimentação no tempo de sedimentação $t$. Com esta velocidade média, a altura da linha de sedimentação pode ser calculada facilmente por

$$
\mathrm{h}(\mathrm{t})=\overline{\mathrm{V}}_{\mathrm{t}} \mathrm{t}
$$

As Equações 5 e 6 podem ser utilizadas para calcular a altura da linha de sedimentação em qualquer tempo $t$, ० que permite reconstruir o ensaio de sedimentação a partir da equação estendida de Wilhelm-Naide. Para tanto, é necessário substituir a Equação do modelo em (3) na Equação 5.

$$
\overline{\mathrm{V}}_{\mathrm{t}}=\frac{\int_{\mathrm{C}(0)}^{\mathrm{C}(\mathrm{t})} \frac{\mathrm{V}_{\mathrm{TF}}}{1+\alpha_{1} \mathrm{C}^{\beta_{1}}+\alpha_{2} \mathrm{C}^{\beta_{2}}+\ldots} \mathrm{dC}}{\mathrm{C}(\mathrm{t})-\mathrm{C}(0)}
$$

A Equação 7 permite estimar os parâmetros $\alpha_{i}$ e $\beta_{i}$ a partir de ensaios de sedimentação utilizando-se uma técnica de otimização acoplada com uma função objetivo de forma conveniente.

A função objetivo utilizada aqui é a soma normalizada dos resíduos em $t$ :

$$
\phi=\frac{\left(t_{j}-\hat{t}_{j}\right)^{2}}{t_{j}^{2}}
$$

onde $\phi$ é o valor da função objetivo, a ser minimizada, $t_{j}$ é o j-ésimo tempo de sedimentação medido no ensaio de sedimentação e $\hat{t}_{\mathrm{j}}$ é o tempo de sedimentação calculado na concentração (ou altura) correspondente, ou seja

$$
\hat{\mathrm{t}}_{\mathrm{j}}=\frac{\mathrm{h}(\mathrm{t})}{\overline{\mathrm{V}}_{\mathrm{t}}}=\frac{\mathrm{h}(\mathrm{t})(\mathrm{C}(\mathrm{t})-\mathrm{C}(0))}{\int_{\mathrm{C}(0)}^{\mathrm{C}(\mathrm{t})} \frac{\mathrm{v}_{\mathrm{TF}}}{1+\alpha_{1} \mathrm{C}^{\beta_{1}}+\alpha_{2} \mathrm{C}^{\beta_{2}}+\ldots} \mathrm{dC}}
$$

A integração na Equação 9 pode ser feita numericamente pelo método de Romberg. A rotina de otimização utilizada aqui é a de Rosenbrock, que não requer derivadas relativas aos parâmetros $\alpha_{i}$ e $\beta_{i}$, facilitando em muito a implementação do sistema.

\section{TRABALHO EXPERIMENTAL}

O trabalho experimental consistiu na amostragem de um espessador industrial de lamas, seguido de testes de sedimentação em proveta. ${ }^{(3)}$

Os dados operacionais do espessador da Usina de Timbopeba são apresentados de forma resumida na Tabela I.

Tabela I. Dados operacionais do espessador.

\begin{tabular}{lcc}
\hline \multicolumn{1}{c}{ Variável } & Valor & Unidade \\
\hline Diâmetro & $6 \mathrm{I}$ & $\mathrm{m}$ \\
Densidade do underflow & $\mathrm{I}, 45$ & $\mathrm{~g} / \mathrm{cm}^{3}$ \\
Sólidos do underflow & 40 & $\%$ \\
Taxa de alimentação de sólidos & 70.9 & $\mathrm{t} / \mathrm{h}$ \\
\hline
\end{tabular}

Foram realizados ensaios de sedimentação variando-se a concentração de floculante entre $0 \mathrm{~g} / \mathrm{t}$ e $25 \mathrm{~g} / \mathrm{t}$, com 6 dosagens distintas. Estes dados foram utilizados para o cálculo dos parâmetros do modelo de Wilhelm-Naide, utilizando-se 
- aplicativo SedimenCalc ${ }^{T M}$ desenvolvido neste trabalho. Para cada dosagem de floculante, foram calculados inicialmente os valores de $\alpha_{1}, \beta_{1}$ e $v_{\text {tf }}$ Esta forma mais simples do modelo, com somente um termo, produz resultados muito satisfatórios, embora o sistema implementado permita a utilização de até três termos. Os resultados para um termo são mostrados na Tabela 2, em unidades do sistema SI.

Tabela 2. Resultados do modelamento dos ensaios de sedimentação.

\begin{tabular}{ccccc}
\hline $\begin{array}{c}\text { Concentração } \\
\text { de floculante } \\
(\mathrm{g} / \mathrm{t})\end{array}$ & $v_{\mathrm{tf}}(\mathrm{mm} / \mathrm{s})$ & $\alpha_{\mathrm{l}}$ & $\beta_{\mathrm{I}}$ & $\begin{array}{c}\text { Função } \\
\text { objetivo } \phi\end{array}$ \\
\hline 0 & 0,670506 & $2,011345 \mathrm{e}-014$ & 5,910776 & 0,132114 \\
5 & 3,452446 & $1,065414 \mathrm{e}-012$ & 5,533600 & 0,143685 \\
10 & 8,400432 & $2,208778 \mathrm{e}-015$ & 6,676480 & 0,291623 \\
15 & 14,049845 & $3,009228 \mathrm{e}-016$ & 7,118387 & 0,667066 \\
20 & 24,111096 & $1,834411 \mathrm{e}-014$ & 6,453715 & 0,227177 \\
25 & 33,962524 & $1,499407 \mathrm{e}-017$ & 7,698754 & 0,046927 \\
\hline
\end{tabular}

Observa-se que os valores da função objetivo são baixos, indicando uma boa qualidade de interpolação. Isto é um bom indicativo de que os ensaios, de forma geral, foram executados de forma satisfatória. A correlação entre $v_{t f}$ e a dosagem de floculante é bastante direta, indicando que o tamanho dos flocos aumenta monotonicamente com a concentração de floculante, e que os flocos são estáveis (não quebram ou desagregam) até $25 \mathrm{~g} / \mathrm{t}$.

A qualidade das soluções mostradas na Tabela 2 é mais bem visualizada graficamente. Isto é ilustrado na Figura $I$.

Claramente, os resultados acima indicam que o modelo com apenas um termo é adequado e que as ferramentas desenvolvidas estão funcionando de forma adequada.

\section{I Escalonamento do Espessador Baseado no Método de Kynch}

Um espessador cilíndrico ideal opera de modo contínuo, com a polpa alimentada abaixo da superfície, com uma interface nítida desenvolvida no nível da alimentação, e o líquido clarificado sobrenadante, para uma concentração de polpa $C_{L}$. Assume-se que a polpa alimentada é espalhada, instantaneamente, na superfície transversal do espessador atingindo uma concentração diluindo-se na concentração $C_{L} \cdot{ }^{(4)}$

Obviamente esta é uma idealização do comportamento de um espessador real. No entanto, esta idealização produz um modelo útil para simulação. Mais abaixo, no espessador, uma interface com concentração $C_{m}$ se desenvolve e, ao fundo, a ação mecânica do rake move a polpa sedimentada para dentro e a polpa espessada é descartada através do underflow com uma concentração $C_{D}$. A concentração de sólidos abaixo da interface de polpa clarificada não é uniforme e aumenta com a profundidade devido à compressibilidade da polpa.

O fluxo de sedimentação total relativo a uma coordenada fixa em qualquer nível onde a concentração é $C$ deve incluir o fluxo volumétrico total devido à remoção da polpa espessada pelo underflow, além do fluxo de sedimentação dos sólidos. Se o fluxo total é representado por $\mathrm{f}(\mathrm{C})$ e o fluxo volumétrico de polpa abaixo da linha de alimentação por $\mathrm{q} \mathrm{m}^{3} / \mathrm{m}^{2}$, então:
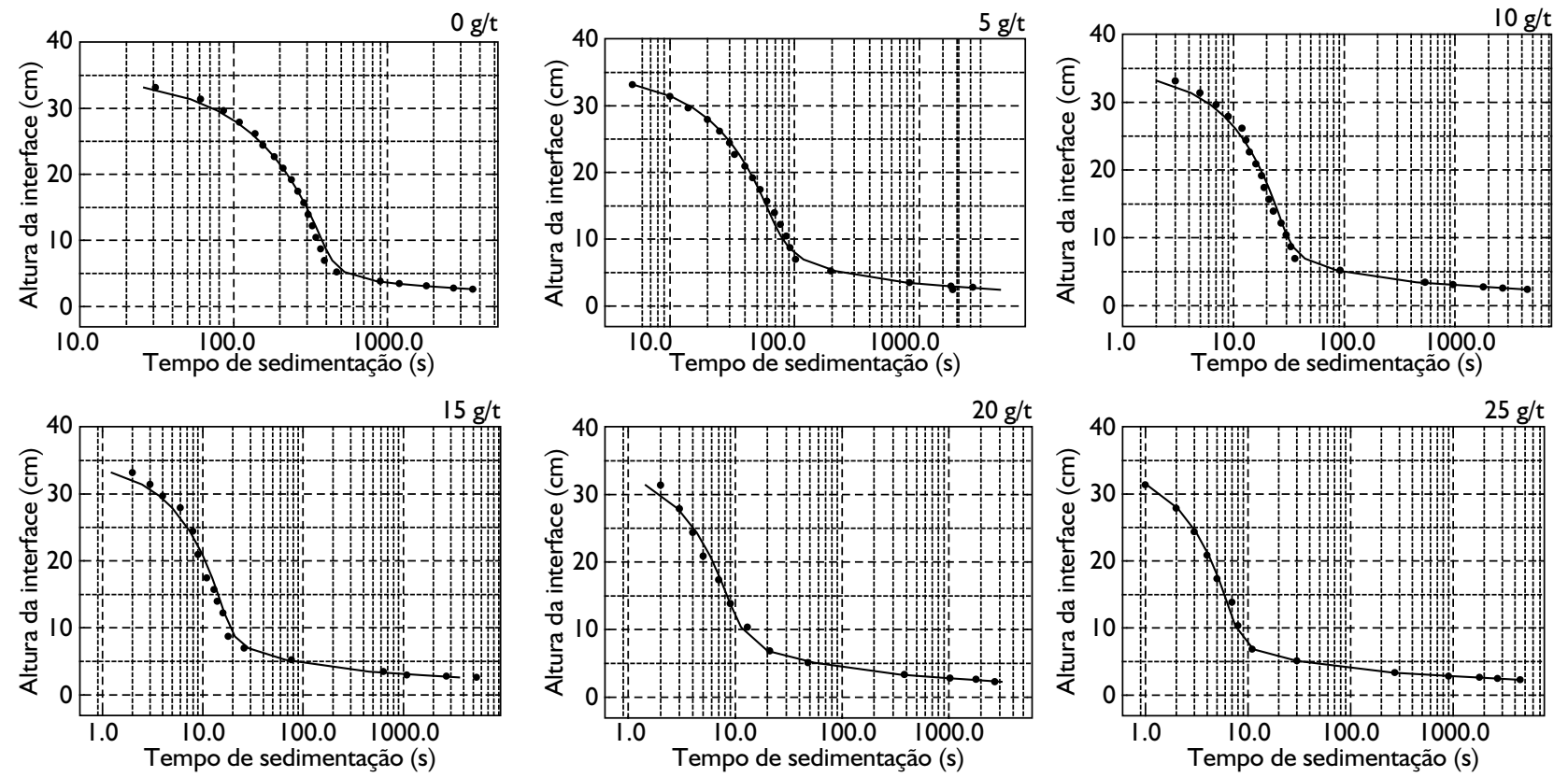

Figura I. Gráficos de altura da interface, em cm, versus tempo de sedimentação, em s. Resultados dos testes mostrados como círculos e a interpolação com o modelo de Wilhelm-Naide com os parâmetros da Tabela 2. 


$$
\mathrm{f}(\mathrm{C})=\mathrm{qC}+\psi(\mathrm{C}) \quad \mathrm{kg} / \mathrm{m}^{2} \mathrm{~s}
$$

Em sedimentação em proveta, $\mathrm{q}=0$ e portanto $\mathrm{f}(\mathrm{C})$ e $\psi(\mathrm{C})$ são idênticos. Uma expressão que descreve a taxa de movimento de uma interface ou descontinuidade em um espessador contínuo pode ser derivada a partir da análise do ensaio de sedimentação em proveta

$$
\sigma\left(\mathrm{C}^{+}, \mathrm{C}^{-}\right)=-\frac{\mathrm{f}\left(\mathrm{C}^{+}\right)-\mathrm{f}\left(\mathrm{C}^{-}\right)}{\mathrm{C}^{+}-\mathrm{C}^{-}} \quad \mathrm{m} / \mathrm{s}
$$

onde $\mathrm{C}^{+}$e $\mathrm{C}^{-}$representam as concentrações de sólidos acima e abaixo da descontinuidade, respectivamente.

Se o espessador está operando em regime contínuo as descontinuidades não se movem (são estacionárias) e $\sigma\left(C^{+}, C^{-}\right)$ deve ser igual a zero em cada descontinuidade. $O$ modelo de um espessador contínuo é baseado no requerimento de que, em regime contínuo e estável, todo o sólido necessariamente passa por todos os planos horizontais no espessador.

O fluxo através de qualquer plano horizontal em um espessador em regime contínuo estável deve ser exatamente igual ao fluxo de alimentação

$$
\mathrm{f}_{\mathrm{F}}=\frac{\mathrm{Q}_{\mathrm{F}} \mathrm{C}_{\mathrm{F}}}{\mathrm{A}}
$$

e o fluxo do underflow

$$
\mathrm{f}_{\mathrm{D}}=\frac{\mathrm{Q}_{\mathrm{D}} \mathrm{C}_{\mathrm{D}}}{\mathrm{A}}=\mathrm{qC}_{\mathrm{D}}
$$

onde $q=Q_{D} / A$ é o fluxo volumétrico total em qualquer leito horizontal abaixo da linha de alimentação. Portanto

$$
f(C)=q C+\psi(C)=f_{F}
$$

onde $C$ é a concentração em qualquer nível onde existam condições de sedimentação livre no espessador.

A Equação 14 pode ser plotada na curva de fluxo versus concentração como uma linha reta de inclinação -q. Isso é mostrado na Figura 2. A linha intercepta o eixo da concentração onde $C=C_{D}$.

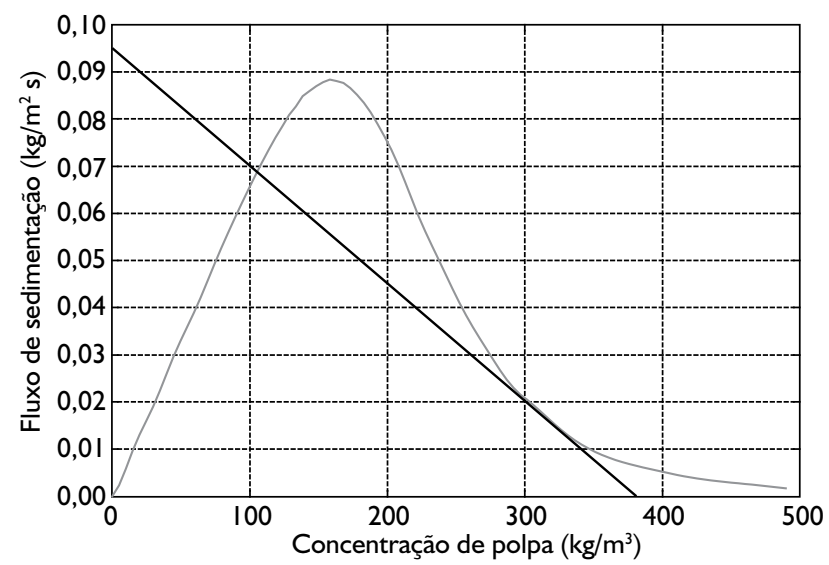

Figura 2. Fluxo de sedimentação em $\mathrm{kg} / \mathrm{m}^{2} \mathrm{~s}$ versus concentração de polpa, em kg/m ${ }^{3}$. Resultado correspondente ao teste de sedimentação com $0 \mathrm{~g} / \mathrm{t}$ de floculante, ilustrando o cálculo de capacidade e concentração no underflow de um espessador imaginário.
Um modelo de espessador simples pode ser construído para o espessador cilíndrico contínuo usando este modelo ideal. Se a área do espessador é dada e as condições da polpa de alimentação conhecidas, então:

$$
\mathrm{f}_{\mathrm{F}}=\mathrm{Q}_{\mathrm{F}} \mathrm{C}_{\mathrm{F}}
$$

Portanto a concentração máxima de polpa no underflow é dada pela intersecção da linha da Figura 2 com o eixo C. O fluxo máximo de alimentação é fixo pela inclinação da curva de fluxo no ponto de inflexão. O método requer um modelo para a curva de fluxo, e o modelo de Wilhelm-Naide pode ser utilizado para um modelo de simulação consistente para o espessador. Quando o espessador é alimentado a uma taxa menor que a máxima, a concentração no underflow pode ser calculada pela intersecção com o eixo horizontal da linha operacional que passa pelo fluxo de alimentação no eixo vertical e que é tangente à curva de fluxo, como mostrado na Figura 2.

Este modelo foi implementado no simulador de plantas Modsim ${ }^{\mathrm{TM}}$, como modelo KYNC. Para as condições operacionais da amostragem do espessador de Timbopeba, foi obtida uma polpa com $44,4 \%$ de sólidos no produto, sem contaminação de sólidos no overflow.

A metodologia desenvolvida representa um avanço na aplicação prática de modelamento de ensaios de sedimentação em proveta e a utilização dos resultados no escalonamento e na simulação de espessadores industriais, através da utilização dos aplicativos Modsim ${ }^{\mathrm{TM}}$ e o aplicativo SedimenCalc ${ }^{T M}$.

Embora a simulação tenha previsto um underflow com $44 \%$ de sólidos contra $40 \%$ de sólidos medidos na planta, estes valores estão dentro da faixa de segurança de escalonamento, e do erro esperado pelo método de Kynch, considerando-se todas as simplificações envolvidas no modelo, e as dificuldades experimentais dos testes de sedimentação em proveta. No entanto, a previsão de uma concentração no underflow maior do que aquela obtida industrialmente, é surpreendente, já que o sistema não contém qualquer disposição que permita levar em conta a compressibilidade da polpa.

Estas interfaces ora implantadas no CDMCVRD facilitam em muito a aplicação do método de escalonamento de espessadores de Kynch, e viabilizam a avaliação de performance de espessadores industriais existentes. 


\section{REFERÊNCIAS}

I WILHELM, J.H.; NAIDE, Y. Sizing and operating continuous thickeners. Mining Engineering, v.33, n. 12, p. 17/0-8, Dec. 1981 .

2 KYNCH, G.J. Theory of sedimentation. Transactions Faraday Society, v. 48, p.166-76, 1952.

3 ALVES, V.K.; GOBBO, O.; GONÇALVES, K. Ensaios de sedimentação em proveta com amostra de alimentação do espessador de lama da Usina de Timbopeba. Belo Horizonte: Companhia Vale do Rio Doce, 2004. [Relatório interno]

4 KING, R.P. Modeling \& simulation of mineral processing systems. Oxford: Butterworth-Heinemann, 200 I.

Recebido em: 25/0I/07

Aceito em: 29/10/07

Proveniente de: SIMPÓSIO BRASILEIRO DE MINÉRIO DE FERRO, 7., 2006, Ouro Preto, MG. São Paulo: ABM, 2006. 